From The University of Texas M. D. Anderson Cancer Center; The University of Texas Graduate School of Biomedical Sciences at Houston, Houston, TX; and the National Cancer Institute, Bethesda, MD.

Submitted May 25, 2010; accepted July 26, 2010; published online ahead of print at www.jco.org on September 20, 2010

Supported by Grant No. N01 CM-62202 from the National Cancer Institute's Cancer Therapy Evaluation Program. Support for correlative laboratory work was provided by The University of Texas Lung Specialized Program of Research Excellence, Bristol-Myers Squibb, and the Commonwealth Foundation for Cancer Research. Dasatinib was provided by Bristol-Myers Squibb and the National Cancer Institute.

Authors' disclosures of potential conflicts of interest and author contributions are found at the end of this article.

Clinical Trials repository link available on JCO.org.

Corresponding author: Faye $\mathrm{M}$. Johnson, MD, PhD, Department of Thoracic/Head and Neck Medical Oncology, Unit 432, The University of Texas M. D. Anderson Cancer Center, 1515 Holcombe Blvd, Houston, TX 770304009; e-mail: fmjohns@mdanderson.org.

(c) 2010 by American Society of Clinical Oncology

0732-183X/10/2830-4609/\$20.00

DOI: $10.1200 / J C O .2010 .30 .5474$

\title{
Phase II Study of Dasatinib in Patients With Advanced Non-Small-Cell Lung Cancer
}

Faye M. Johnson, B. Nebiyou Bekele, Lei Feng, Ignacio Wistuba, Xi Ming Tang, Hai T. Tran, Jeremy J. Erasmus, Li-Ling Hwang, Naoko Takebe, George R. Blumenschein, Scott M. Lippman, and David J. Stewart

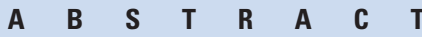

\section{Purpose}

Src family kinases (SFKs) promote cancer progression and are commonly expressed in non-smallcell lung cancer (NSCLC), but the clinical effects of SFK inhibition in NSCLC are unknown. We conducted a phase II trial of the SFK inhibitor dasatinib for advanced NSCLC. We tested the hypotheses that the activation of epidermal growth factor receptor (EGFR) or SFK or modulation of serum cytokines may predict a response to dasatinib.

\section{Patients and Methods}

Patients received dasatinib as first-line therapy. Response was measured by tumor size on computed tomography scans and by metabolic activity on positron emission tomography scans. Tissue samples taken before patients received dasatinib were tested for EGFR and Kras mutation and phosphorylated SFK expression.

\section{Results}

Thirty-four patients were enrolled. The overall disease control rate (partial responses plus stable disease) for dasatinib was $43 \%$. One patient had a partial response to therapy. Eleven patients (32\%) had a metabolic response to dasatinib. SFK activation and EGFR and Kras mutations in tumor tissue did not predict response to dasatinib. Significant toxicities included fatigue and dyspnea. The presence of a pleural effusion before dasatanib therapy predicted the development of a clinically significant effusion during therapy.

\section{Conclusion}

Dasatinib as a single agent had modest clinical activity that was lower than that generally observed in patients with NSCLC who receive chemotherapy. Pleural effusion was an expected and problematic toxicity that was successfully treated with steroids, diuretics, and dose interruptions. Marked activity in one patient and prolonged stable disease in four others suggested a potential subpopulation of patients with dasatinib-sensitive NSCLC.

\section{J Clin Oncol 28:4609-4615. (C) 2010 by American Society of Clinical Oncology}

\section{INTRODUCTION}

Non-small-cell lung cancer (NSCLC) is a lethal disease for which improved systemic therapy is desperately needed. The Src family kinases (SFKs) are nonreceptor tyrosine kinases involved in signal transduction that are potential therapeutic targets in NSCLC. SFK inhibition leads to decreased angiogenesis, invasion, proliferation, and survival of cancer cells. ${ }^{1,2}$ Activated SFKs are commonly expressed by NSCLC. ${ }^{3,4}$

SFK inhibition universally inhibits invasion by NSCLC cells but has variable effects on apoptosis., Inhibiting SFKs in NSCLC cell lines that have activating epidermal growth factor receptor (EGFR) mutations leads to significant apoptosis. ${ }^{4-10}$ However, apoptosis is rarely observed in NSCLC cell lines that have wild-type EGFR. ${ }^{5}$ In addition to its direct antitumor effects, SFK inhibition also inhibits angiogenesis via effects on the production of proangiogenic factors. ${ }^{11-14}$ Whether these laboratory findings translate into the clinical setting is not known.

Dasatinib is a potent SFK inhibitor that may have a clinical benefit in patients with NSCLC. However, patients with NSCLC often have preexisting lung disease that may adversely affect their ability to tolerate dasatinib, which often causes pleural effusion. ${ }^{15-17}$

We conducted a phase II study of dasatinib as first-line treatment for metastatic NSCLC. The primary objective of this trial was to determine the rate of progression-free survival (PFS) at 12 weeks. Secondary objectives included measuring metabolic response and toxicity; examining the relationship 
between clinical response to dasatinib and EGFR mutational status, EGFR copy number, and activated SFK expression levels in pretreatment tumor biopsies; and determining whether correlations exist between clinical response to dasatinib and drug exposure, pharmacodynamics (ie, phosphorylated Src [pSrc] levels in platelets), or changes in serum levels of several cytokines. We hypothesized that patients with NSCLC who had baseline activation of SFKs or the EGFR pathway are more likely to respond to dasatinib and that responders are more likely to demonstrate higher drug exposure and have a reduction in proangiogenic cytokine levels during therapy.

\section{PATIENTS AND METHODS}

\section{Eligibility Criteria}

Adults with stage IV, radiographically measurable NSCLC and an Eastern Cooperative Oncology Group (ECOG) performance status of 0 or 1 were eligible to participate. Patients who had previously been treated with systemic therapy for metastatic NSCLC were excluded; prior systemic therapy as part of potentially curative treatment was acceptable. Additional eligibility criteria included a neutrophil count $>3,000$ cells $/ \mu \mathrm{L}$, a platelet count $>100,000$ cells $/ \mu \mathrm{L}$, a bilirubin level $<1.5 \times$ the upper limit of normal (ULN) for the institution, a creatinine level $<3 \times$ the ULN, and transaminase level $<2.5 \times$ the ULN. Patients with serious concurrent medical illness were excluded. All patients were required to have pretreatment tumor tissue specimens available for analysis before enrollment. For the majority of patients, the existing tissue blocks were deemed adequate, and only one protocol-specific biopsy was required. Institutional review board approval was obtained, and patients gave written informed consent.

\section{Study Design and Treatment}

This was a single-arm, open-label study in which all patients received oral dasatinib daily until disease progression, unacceptable toxicity, or withdrawal of consent. The first 22 patients began treatment with $100 \mathrm{mg}$ twice a day. Because of poor tolerance (fatigue and pleural effusion), the remaining 11 patients began treatment with $100 \mathrm{mg}$ in the morning and $50 \mathrm{mg}$ in the evening. Blood for optional pharmacokinetic, pharmacodynamic, and cytokine profiles was drawn at baseline and on day 21 .

\section{Assessment of Clinical Activity}

$\left[{ }^{18} \mathrm{~F}\right]$ fluorodeoxyglucose positron emission tomography/computed tomography (FDG-PET/CT) was performed at baseline and at 6 and 12 weeks after initiation of dasatinib; CT was performed every 6 weeks thereafter. Response was assessed by using Response Evaluation Criteria in Solid Tumors (RECIST). ${ }^{18}$ Disease control rate was defined as partial response (PR) plus stable disease (SD). Two-dimensional PET and non-contrast-enhanced CT images were acquired from the base of the skull to the pelvis (speed, 13.5 $\mathrm{mm}$ /rotation; slice thickness, $3.75 \mathrm{~mm} ; 140 \mathrm{kVp} ; 120 \mathrm{~mA}$ ) by using a GE Discovery RX PET/CT scanner (GE Healthcare, Waukesha, WI) 90 minutes after intravenous administration of 300 to $450 \mathrm{MBq}$ FDG. Data were corrected for attenuation, random scatter, and dead time and were reconstructed by using an ordered subsets expectation maximization algorithm. PET images were analyzed on an Xeleris workstation (GE Medical Systems, Milwaukee, WI). FDG uptake was considered visually abnormal if there was more than mediastinal blood-pool activity. Regions of increased FDG uptake were outlined by using a pixel region of interest and were semiquantitatively analyzed by using standardized uptake ratio $\left(\mathrm{SUV}_{\max }\right)$ equal to mean region of interest activity in millicuries per milliliter per injected dose in millicuries per body mass (in grams). $\mathrm{SUV}_{\max }$ was determined for up to five lesions with the most intense FDG uptake. Response to therapy was assessed by using modified PET Response Criteria in Solid Tumors criteria. ${ }^{19}$ A complete metabolic response was defined as the resolution of all metabolically active tumors, a partial metabolic response (PMR) as $>25 \%$ decrease in the $\mathrm{SUV}_{\max }$ of the most FDG-intense lesion, and progressive metabolic disease (PMD) as $>$ a $25 \%$ increase in the $\mathrm{SUV}_{\max }$ or appearance of new lesions. All other outcomes were classified as stable metabolic disease (SMD). Laboratory and statistical methods are provided in the Appendix (online only).

\section{RESULTS}

\section{Patient Characteristics}

The baseline characteristics of the 34 patients enrolled are presented in Table 1. Seven patients had received cytotoxic chemotherapy as part of prior potentially curative therapy. No patients received prior targeted therapy.

\section{Treatment}

The median treatment duration was 1.36 months (range, 0.16 to 17.2 months). The median daily dasatinib dose was $178 \mathrm{mg}$. Twentysix patients discontinued dasatinib treatment because of progressive disease (RECIST), three because of comorbid conditions unrelated to therapy, and five because of toxicity that was likely dasatinib-related (grade 2 fatigue, grade 3 dehydration, and grade 2 headache).

\section{Response and Survival}

Four patients who discontinued dasatinib because of toxicity or unrelated medical problems were not evaluable for response. At 12

\begin{tabular}{|c|c|c|}
\hline Characteristic & $\begin{array}{l}\text { No. of Patients } \\
(n=34)\end{array}$ & $\%$ \\
\hline \multicolumn{3}{|l|}{ Age, years } \\
\hline Median & \multicolumn{2}{|l|}{69} \\
\hline Range & \multicolumn{2}{|l|}{$51-79$} \\
\hline \multicolumn{3}{|l|}{ Performance status } \\
\hline 0 & 10 & 29 \\
\hline 1 & 24 & 71 \\
\hline \multicolumn{3}{|l|}{ Sex } \\
\hline Female & 10 & 29 \\
\hline Male & 24 & 71 \\
\hline \multicolumn{3}{|l|}{ Ethnicity } \\
\hline Asian & 1 & 3 \\
\hline Black & 2 & 6 \\
\hline Hispanic & 2 & 6 \\
\hline White & 29 & 85 \\
\hline \multicolumn{3}{|l|}{ Histology } \\
\hline Adenocarcinoma & 25 & 74 \\
\hline Squamous cell carcinoma & 6 & 18 \\
\hline Poorly differentiated NSCLC & 3 & 9 \\
\hline \multicolumn{3}{|l|}{ EGFR status } \\
\hline Wild type & 22 & 65 \\
\hline Classic mutation (L858R or exon 19 del) & 6 & 18 \\
\hline Other mutation & 3 & 9 \\
\hline No data & 3 & 9 \\
\hline \multicolumn{3}{|l|}{ Kras status } \\
\hline Wild-type & 24 & 71 \\
\hline Mutant & 7 & 21 \\
\hline No data & 3 & 9 \\
\hline \multicolumn{3}{|l|}{ Prior therapy } \\
\hline None & 21 & 62 \\
\hline Surgery and/or radiotherapy & 6 & 18 \\
\hline Concurrent chemotherapy and radiotherapy & 4 & 12 \\
\hline Adjuvant chemotherapy & 3 & 9 \\
\hline
\end{tabular}

Abbreviations: NSCLC, non-small-cell lung cancer. 
weeks, one patient had a $\mathrm{PR}, 12$ patients had $\mathrm{SD}$, and 17 patients had progressive disease as defined by RECIST (Appendix Fig A1, online only). The overall disease control rate was $43 \%$. The median PFS was 1.36 months (Fig 1A), and the median overall survival was 11.4 months (Fig 1B). The difference in PFS between male and female patients was not significant $(P=.80$; log-rank test).

Of the 21 patients who were evaluable for metabolic response to dasatinib, seven had PMR, eight had SMD, and six had PMD (Appen-

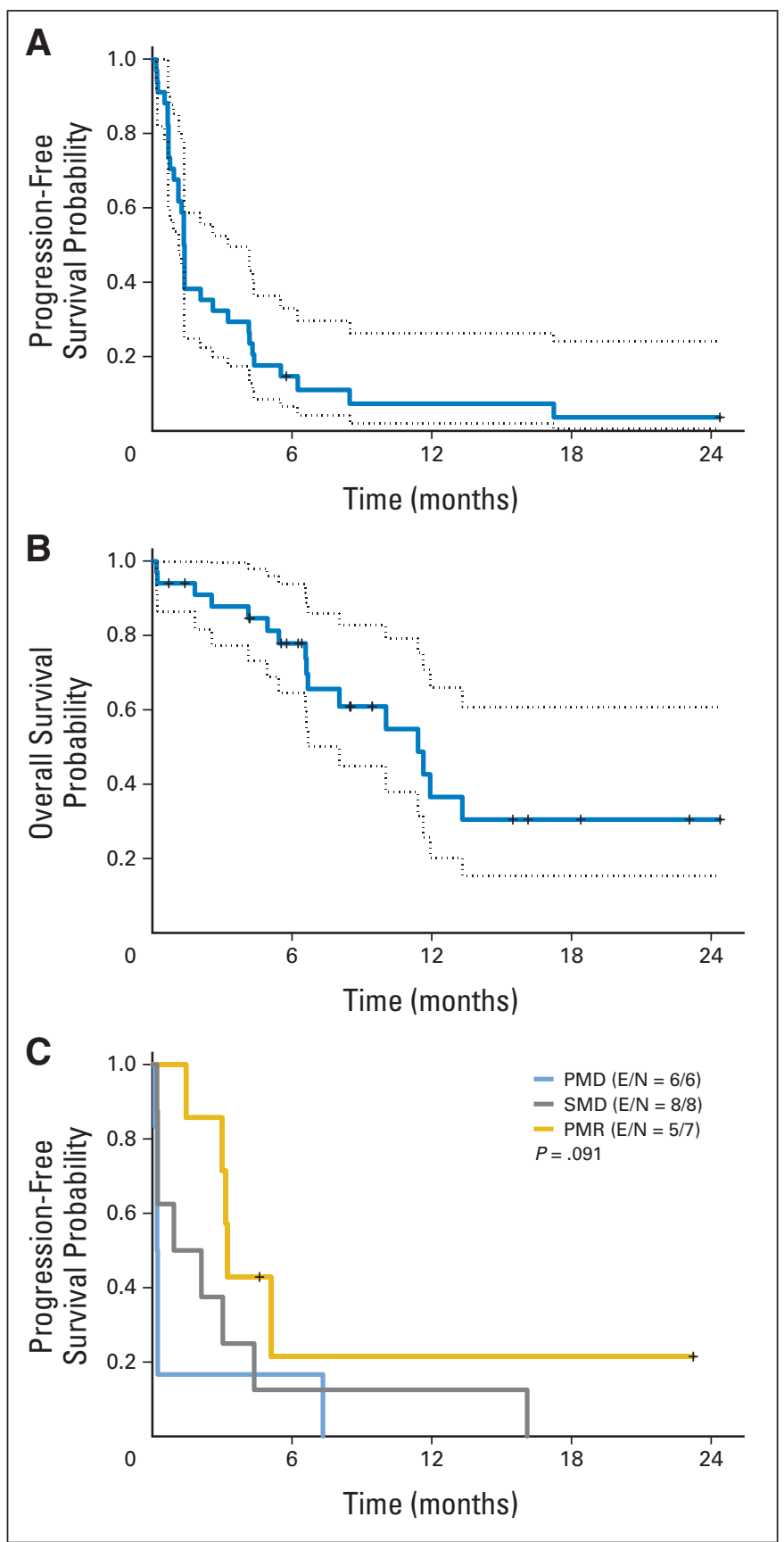

Fig 1. Clinical response to dasatinib. (A) Progression-free survival probability (blue line). Dashed lines represent the $95 \% \mathrm{Cls}$. (B) Overall survival probability (blue line). Dashed lines represent the $95 \% \mathrm{Cls}$. (C) Progression-free survival probability for patients with progressive metabolic disease (PMD), stable metabolic disease (SMD), and partial metabolic response (PMR). E/N, number of events/total number of evaluable patients. $\operatorname{dix}$ Fig A2, online only). Patients with PMD tended to have a shorter PFS $(P=.091$; Fig $1 C)$. There was no significant association between sex and metabolic response.

Four patients had SD for 6 months or longer. The only patient who had a PR was a former smoker (38 pack-years) with adenocarcinoma with wild-type EGFR and a Kras polymorphism (Appendix Fig A3, online only). He discontinued dasatinib after 12 weeks because of malaise and did not receive any other therapy. PET/CT revealed a complete metabolic response at 7 months. At 28 months his only detectable NSCLC is a non-metabolically active 1-cm lung nodule (Fig 2).

Because of the important role of c-Src signaling in bone physiology, we examined the initial appearance, FDG uptake, and changes in bone metastasis after therapy. Seven patients (33\%) had bone metastases. The bone metastases were predominantly lytic $(n=6)$ and all were FDG-avid ( $\mathrm{SUV}_{\max }$ range, 3.5 to 23.5; mean, 9.7) on baseline PET/CT. Four bone metastasis patients had repeat PET/CT at 6 weeks and three of those had a PMR and one had SMD. Three patients had PET/CT evaluation at 12 weeks with one having PMR and two having SMD. All the bone metastases were unchanged in size and appearance on CT.

\section{Toxicity}

Toxicity findings (Table 2) were consistent with those in patients who received dasatinib for other solid tumors. ${ }^{15,17,20,21}$ Significant laboratory abnormalities were rare. The most common grade 3 toxicity was dyspnea caused by underlying chronic obstructive pulmonary disease, thoracic tumors, and/or pleural effusion. Mild nausea with associated dehydration was a common, mild, and expected toxicity. Grade 3 pain occurred in two patients and included dasatinib-related headache and pleuritic chest pain. Ten patients had dose reductions (eight for dyspnea/pleural effusion, one for neutropenia, and one for fatigue), and 15 patients had dasatinib held during the study (11 for dyspnea/pleural effusion, one for neutropenia, and three for respiratory infections).

Two patients died during the study. Neither death was considered related to dasatinib therapy. One patient was admitted to the hospital after 4 days of dasatinib therapy. Aggressive therapy was not pursued and he died 2 days later, likely as a result of cancer progression. The second patient died in his sleep after receiving dasatinib for only 2 days; no autopsy was performed.

Because pleural effusions are common in patients who receive dasatinb, we examined factors that may predict this toxicity. Patients with a pre-existing pleural effusion were at a higher risk of developing a high-grade, clinically significant effusion during dasatinib therapy than patients who did not have a pre-existing pleural infusion $(P=.0017)$. Aggressively managing pleural effusions with treatment interruptions, diuretics, and steroids was successful in most patients.

The starting dose of the trial was reduced because of poor tolerance (pleural effusion and fatigue). In the patients who received the higher starting dose ( $v$ those who started at the lower dose), new pleural effusions (36\% v 16\%) and grade 3 effusions (23\% v 8\%) were more common, although grade 2 to 3 dyspnea (50\% v 42\%) was not markedly different. Fatigue (grades 2 to 3 ) was more common in the patients who received the higher starting dose $(41 \%)$ compared with those who started at the lower dose $(0 \%)$. 

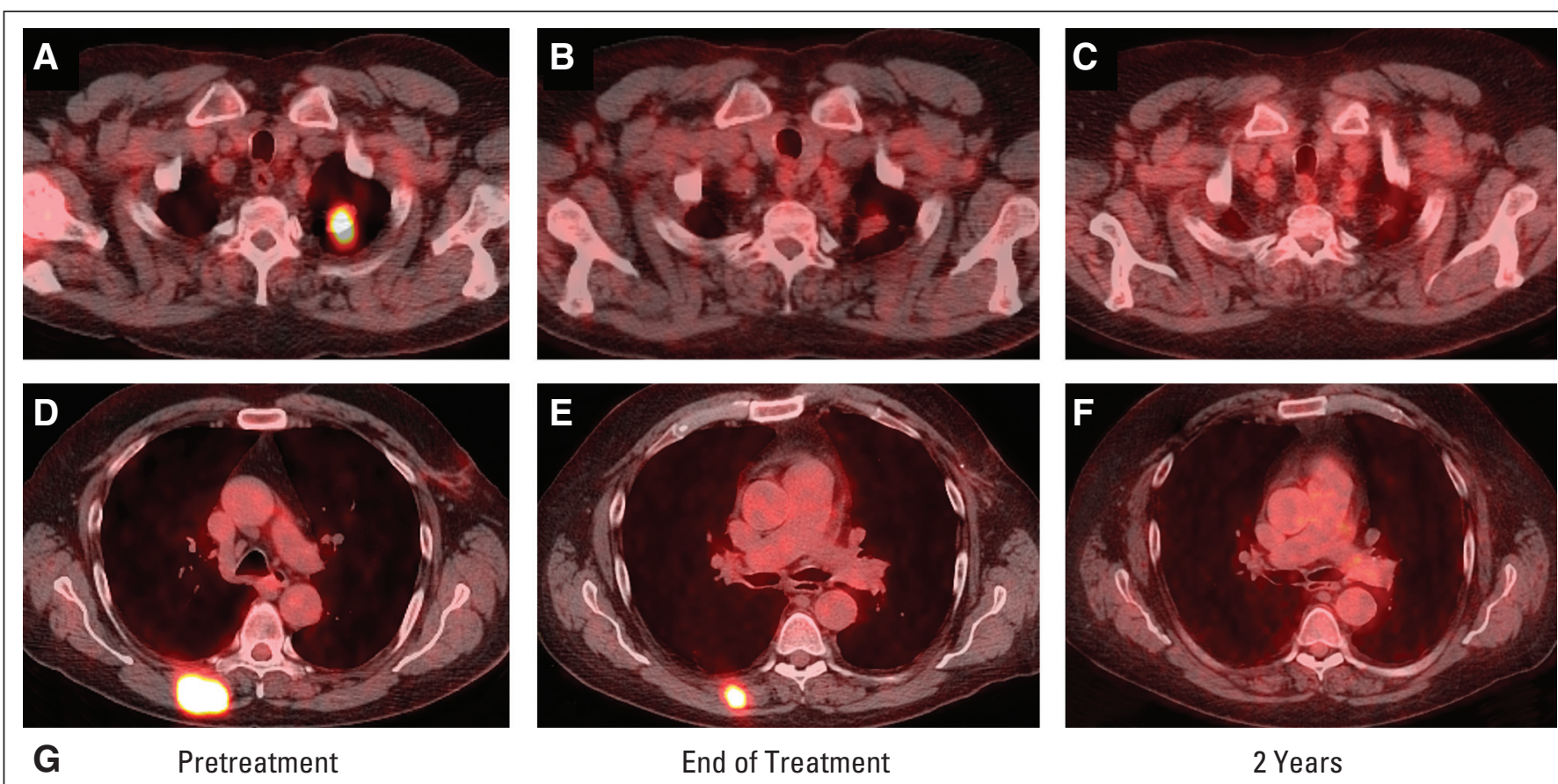

End of Treatment

2 Years

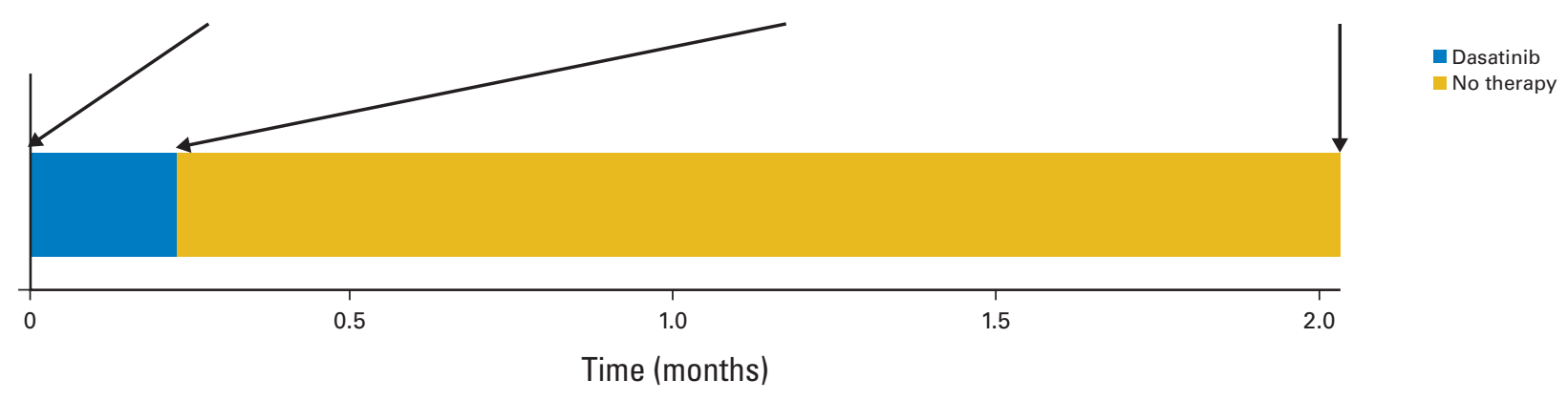

Fig 2. Integrated positron emission tomography/computed tomography scans for one patient before starting dasatinib therapy ( $A, D)$, at the end of therapy ( $B, E)$, and

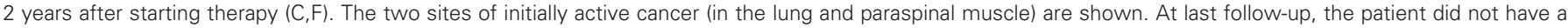
radiographically detectable lesion in the paraspinal muscle. He had a residual non-[ $\left.{ }^{18} \mathrm{~F}\right]$ fluorodeoxyglucose-avid lung nodule.(G) Duration of dasatinib therapy.

\section{Pharmacokinetics, Pharmacodynamics, and Plasma Cytokine Levels}

Nine patients had pretreatment cytokine profiles, and five patients had paired blood samples from before and after treatment. The baseline levels of three cytokines were associated with PFS, but because the hazard ratios (HRs) were all $<1.02$, a clinically significant role for these cytokines in NSCLC response to dasatinib seems unlikely (Appendix Table A1, online only). Cytokine modulation was not associated with PFS in this small patient population (Appendix Table A2, online only).

We also evaluated the cytokine profile in relation to pleural effusion because this toxicity may be immune-mediated. The mean baseline value of osteopontin was $76,531 \mathrm{pg} / \mathrm{mL}$ in patients who did not develop an effusion during dasatinib therapy and 40,320 pg/mL in patients who did $(P=.037)$. Because all patients with paired blood samples developed effusions, an analysis of correlation between effusion development and changes in cytokine expression was impossible.

Pharmacokinetic samples were analyzed and area under the concentration-time curve (AUC) and maximum drug concentration $\left(\mathrm{C}_{\max }\right)$ values were calculated for eight patients (Appendix Table A3, online only). Neither AUC nor $\mathrm{C}_{\max }$ was significantly associated with PFS. Similarly, the initial dasatinib dose was not associated with PFS $(P=.658)$. In fact, the AUC and $C_{\max }$ values were below the mean and median values for the one patient with a PR.

Thirteen patients had pharmacodynamic measurements of pSrc in platelets at baseline, and 11 of these patients had paired studies. In nearly all patients, $\mathrm{pSrc}$ values were significantly lower after treatment (Fig 3). Neither the baseline platelet pSrc (HR, 1.006; $P=.178)$ nor the change in pSrc $(\mathrm{HR}, 4.97 ; P=.426)$ was associated with PFS.

\section{Tumor Characteristics}

EGFR and Kras mutational analyses were performed successfully in 31 tumors (Appendix Table A4, online only); immunohistochemistry and fluorescent in situ hybridization (FISH) were performed in 21 tumors. Baseline EGFR mutational status, Kras mutational status, EGFR amplification, pSrc score (as a continuous or dichotomous variable), phosphorylated signal transducer and activator of transcription 3 (pSTAT3) level, and histology were not significant predictors of PFS $(P=.697, .308, .149, .987, .114$, and .675 , respectively). The four 


\begin{tabular}{|c|c|c|c|c|}
\hline \multirow[b]{2}{*}{ Toxicity } & \multicolumn{2}{|l|}{ Grade 2} & \multicolumn{2}{|l|}{ Grade 3} \\
\hline & No. of Patients & $\%$ & No. of Patients & $\%$ \\
\hline \multicolumn{5}{|l|}{ Pulmonary } \\
\hline Dyspnea & 1 & 3 & 15 & 44 \\
\hline Pleural effusion & 9 & 26 & 6 & 18 \\
\hline Cough & 1 & 3 & 0 & \\
\hline Hypoxia & 1 & 3 & 1 & 3 \\
\hline Pneumonia & 0 & & 1 & 3 \\
\hline \multicolumn{5}{|l|}{ GI } \\
\hline Nausea & 2 & 6 & 0 & \\
\hline Vomiting & 1 & 3 & 0 & \\
\hline Diarrhea & 2 & 6 & 0 & \\
\hline Anorexia & 2 & 6 & 0 & \\
\hline Elevated ALT/AST & 2 & 6 & 0 & \\
\hline Dehydration & 0 & & 1 & 3 \\
\hline \multicolumn{5}{|l|}{ Skin } \\
\hline Rash & 1 & 3 & 1 & 3 \\
\hline Edema & 1 & 3 & 0 & \\
\hline \multicolumn{5}{|l|}{ Hematologic } \\
\hline Thrombocytopenia & 0 & & 0 & \\
\hline Anemia & 1 & 3 & 1 & 3 \\
\hline Neutropenia & 0 & & 1 & 3 \\
\hline Lymphopenia & 5 & 15 & 1 & 3 \\
\hline \multicolumn{5}{|l|}{ Other } \\
\hline Fatigue & 7 & 21 & 2 & 6 \\
\hline Neuropathy & 1 & 3 & 0 & \\
\hline Pain & 0 & & 2 & 6 \\
\hline Atrial fibrillation & 0 & & 1 & 3 \\
\hline
\end{tabular}

"No grade 4 toxicities were observed.

patients who had SD for 6 months or longer all had adenocarcinoma with wild-type EGFR and Kras.

\section{DISCUSSION}

This phase II study of dasatinib demonstrated moderate clinical activity in a molecularly unselected population of patients with NSCLC.

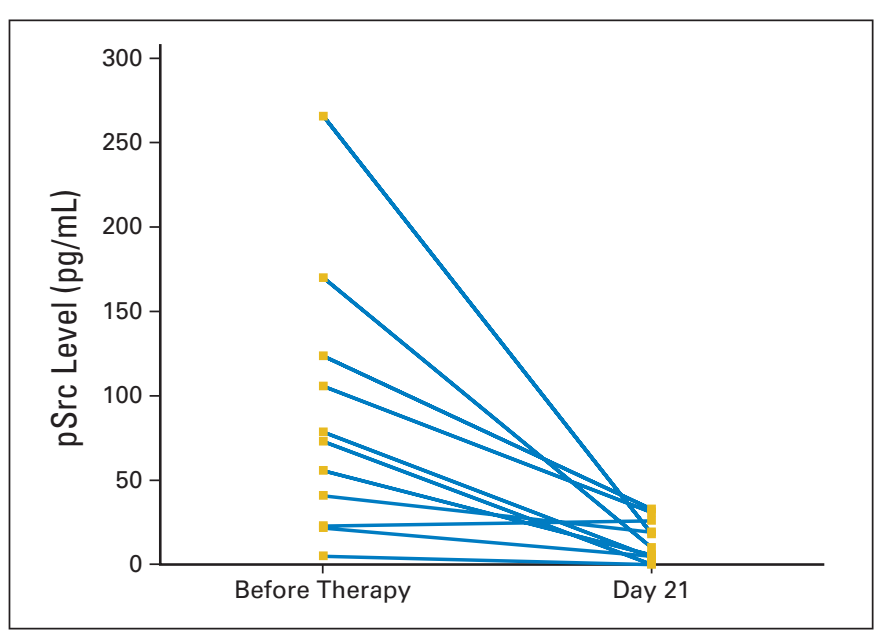

Fig 3. Activated (phosphorylated) Src (pSrc) family kinase level in patients' platelets before starting dasatinib therapy and on day 21 of dasatinib therapy.
Although the marked activity of dasatinib in one patient and prolonged SD in four others suggests a potential subpopulation of patients with dasatinib-sensitive NSCLC, we were unable to identify any biomarkers associated with response to therapy. No grade 4 or unexpected toxicities occurred, ${ }^{15,20}$ but pleural effusion and fatigue were problematic in this population. We found an association between metabolic response on PET images and PFS.

The clinical activity of dasatinib in NSCLC patients did not compare favorably to historical responses to standard therapy. Contemporary studies of standard first-line therapy for NSCLC have demonstrated disease control rates of approximately $40 \%$ and a PFS of 3.6 to 6 months ${ }^{22-24}$ compared with $43 \%$ and 1.36 months in this study. In contrast, the median overall survival of 11.4 months was favorable compared with historical controls. The discrepancy may occur because of unintended patient selection (ie, those patients with more symptomatic, and presumably aggressive, disease would be treated with chemotherapy and those with suspected indolent disease would be treated with dasatinib). Notably, the favorable overall survival suggests that there was no harm in treating patients with an experimental agent as first-line therapy.

Response rates in an unselected population do not always reflect the true clinical utility of targeted agents, as evidenced by the distinct response rates to the EGFR inhibitor erlotinib in patients with NSCLC with $(71 \%)$ and without (1\%) EGFR mutations. ${ }^{24}$ Thus, one goal of this study was to identify biomarkers that predict response to dasatinib. Although several in vitro studies have shown that NSCLCs harboring EGFR mutations were sensitive to the proapoptotic effects of SFK inhibition, this did not translate into a clinical effect in this study. Activated SFK levels did not predict response. ${ }^{5}$ SFK inhibition has variable effects on NSCLC apoptosis in vitro. ${ }^{5,6}$ One potential mechanism for resistance to SFK inhibitors is a lack of sustained STAT3 inhibition that occurs following SFK inhibition. ${ }^{25}$ STAT3 reactivation allows proliferative signals to be maintained despite sustained and significant SFK inhibition.

The clinical activity observed in the current study could have occurred via alternative dasatinib targets such as platelet-derived growth factor receptor (PDGFR) or c-KIT. ${ }^{26}$ Copy number gain in ephrin receptors, Abl, or SFK are factors that affect NSCLC's sensitivity to dasatinib in vitro. ${ }^{27} \mathrm{Li}$ et $\mathrm{al}^{26}$ identified nearly 40 distinct kinase targets of dasatinib, although only a subset was important for NSCLC cytotoxicity in vitro. (These data were published after the patient accrual for this study was completed, and residual patient tissue samples limit further testing.) Rare NSCLC cell lines with increased PDGFR gene copy numbers and hyperphosphorylation are profoundly sensitive to PDGFR inhibitors. ${ }^{28}$ Future studies of dasatinib should incorporate the measurement of gene copy numbers and mutational status for all dasatinib targets.

One patient's marked sensitivity to dasatinib is in keeping with the concept that common tumors may be caused by several different mutations, each of which may require a unique targeted therapy. ${ }^{29}$ The unusual pattern of muscle metastasis in the responding patient suggests that his NSCLC had an unusual biology. One of the few tumors that commonly metastasizes to muscle is melanoma, which frequently harbors activating mutations in c-KIT. ${ }^{30}$ The ligand for c-KIT is stem-cell factor, and high levels of stem-cell factor correlated with prolonged PFS. NSCLC does not commonly express c-KIT ${ }^{31,32}$ nor are mutations or gene amplifications generally found ${ }^{28}$; however, 
this does not rule out c-KIT's role in NSCLC progression in a small subset of patients.

There are several limitations to our study related to the tumor biomarker analysis. In several patients who had received prior therapy for NSCLC, the original diagnostic material was the only tissue available for analysis, and the prior therapy may have affected the biomarkers. In addition, because tumors are heterogeneous, a biopsy specimen may not represent the molecular features of the entire tumor. Discrepancies in mutational status between nodal metastases and primary tumors may also exist. ${ }^{33}$ The effects of prolonged tumor ischemia, as may occur during delays in resection or fixation, may have prevented an accurate analysis of pSrc. ${ }^{34,35}$ Adopting a standard for tissue handling will improve biomarker reliability because tissue handling time before fixation is not routinely recorded, even in prospective studies. ${ }^{36}$

Although single-agent dasatinib in this unselected population of patients with NSCLC was not promising, future studies of combination therapies that include SFK inhibitors are warranted. c-Src can mediate tumor resistance to cytotoxic chemotherapy, and SFK inhibition has been shown to enhance response to cisplatin in vitro in colon and lung cancer cell lines. ${ }^{37,38}$ In addition, SFK inhibition may enhance the antitumor effects of radiotherapy. ${ }^{39}$ Combining SFK inhibitors and EGFR inhibitors increases apoptosis with synergistic and additive effects observed in NSCLC with mutant and wild-type $E G F R$, respectively. ${ }^{2,3}$ In a recently published clinical trial of dasatinib (100 to $140 \mathrm{mg}$ daily) in combination with erlotinib (100 to $150 \mathrm{mg}$ daily) in patients with previously treated NSCLC, a disease control rate of $63 \%$ was achieved and two of 34 patients achieved a PR. ${ }^{21}$ The superior efficacy of the combination in a more refractory patient population demonstrates that the combination is more active than dasatinib as a single agent, confirming the results of preclinical data.,

There was no significant unexpected toxicity in this study. Lowgrade fatigue occurred and caused three patients to withdraw from the study. Fatigue may have been a more clinically significant issue than expected because, unlike the intermittent administration of cytotoxic chemotherapy, dasatinib is given daily, so patients continually experience its adverse effects. Pleural effusion was another expected but problematic toxicity because patients with NSCLC have underlying lung dysfunction and were particularly susceptible to dyspnea. A short course of steroids, the use of diuretics, and treatment interruptions often resolved pulmonary symptoms permanently. ${ }^{16}$ Early and aggressive management of effusions and intermittent dosing of dasatinib should be considered in future clinical studies. ${ }^{20}$ Interestingly, the reported severity of pleural effusions in patients who received the combination of dasatinib and erlotinib was lower than that in patients who received dasatinib alone in this study and in a phase I study of dasatinib in patients with solid tumors. ${ }^{15}$ This may have been because of the aggressive use of steroids in the combination study or it may have been an unexpected, beneficial effect of erlotinib. ${ }^{21}$

To the best of our knowledge, ours is the first study of an SFK inhibitor used alone in patients with NSCLC. We observed clinically significant activity that was unrelated to EGFR status or activated SFK expression in a small number of patients. Our data indicate that using single-agent dasatinib in an unselected population of patients with NSCLC is not warranted. Several patients had metabolic responses that were associated with prolonged PFS, suggesting that PET may play a role in predicting clinical benefit in patients with NSCLC. Future NSCLC studies should focus on defining the mechanisms that lead to enhanced dasatinib sensitivity.

\section{AUTHORS' DISCLOSURES OF POTENTIAL CONFLICTS OF INTEREST}

Although all authors completed the disclosure declaration, the following author(s) indicated a financial or other interest that is relevant to the subject matter under consideration in this article. Certain relationships marked with a " $U$ " are those for which no compensation was received; those relationships marked with a "C" were compensated. For a detailed description of the disclosure categories, or for more information about ASCO's conflict of interest policy, please refer to the Author Disclosure Declaration and the Disclosures of Potential Conflicts of Interest section in Information for Contributors.

Employment or Leadership Position: None Consultant or Advisory Role: Faye M. Johnson, Bristol-Myers Squibb (C) Stock Ownership: None Honoraria: None Research Funding: None Expert Testimony: None Other Remuneration: None

\section{AUTHOR CONTRIBUTIONS}

Conception and design: Faye M. Johnson, Ignacio Wistuba, Hai T. Tran, David J. Stewart

Administrative support: Li-Ling Hwang

Provision of study materials or patients: Faye M. Johnson, Ignacio Wistuba, Naoko Takebe, George R. Blumenschein, Scott M. Lippman, David J. Stewart

Collection and assembly of data: Faye M. Johnson, Ignacio Wistuba, Xi Ming Tang, Hai T. Tran, Jeremy J. Erasmus, Li-Ling Hwang

Data analysis and interpretation: Faye M. Johnson, B. Nebiyou Bekele, Lei Feng, Jeremy J. Erasmus

Manuscript writing: Faye M. Johnson, B. Nebiyou Bekele, Lei Feng, Ignacio Wistuba, Xi Ming Tang, Hai T. Tran, Jeremy J. Erasmus, Li-Ling Hwang, Naoko Takebe, George R. Blumenschein, Scott M. Lippman, David J. Stewart

Final approval of manuscript: Faye M. Johnson, B. Nebiyou Bekele, Lei Feng, Ignacio Wistuba, Xi Ming Tang, Hai T. Tran, Jeremy J. Erasmus, Li-Ling Hwang, Naoko Takebe, George R. Blumenschein, Scott M. Lippman, David J. Stewart

\section{REFERENCES}

1. Johnson FM, Gallick G: Src Family Kinase Inhibitors in Cancer Therapy. Hauppauge, NY, Nova Science Publishers, 2009

2. Kim LC, Song L, Haura EB: Src kinases as therapeutic targets for cancer. Nat Rev Clin Oncol 6:587-595, 2009

3. Masaki $T$, Igarashi $K$, Tokuda $M$, et al: pp60csrc activation in lung adenocarcinoma. Eur $\mathrm{J}$ Cancer 39:1447-1455, 2003
4. Zhang J, Kalyankrishna S, Wislez M, et al: SRC-family kinases are activated in non-small cell lung cancer and promote the survival of epidermal growth factor receptor-dependent cell lines. Am J Pathol 170:366-376, 2007

5. Johnson FM, Saigal B, Talpaz M, et al: Dasatinib (BMS-354825) tyrosine kinase inhibitor suppresses invasion and induces cell cycle arrest and apoptosis of head and neck squamous cell carcinoma and non-small cell lung cancer cells. Clin Cancer Res 11:6924-6932, 2005

6. Purnell PR, Mack PC, Tepper CG, et al: The Src inhibitor AZD0530 blocks invasion and may act as a radiosensitizer in lung cancer cells. J Thorac Oncol 4:448-454, 2009

7. Song L, Morris M, Bagui $T$, et al: Dasatinib (BMS-354825) selectively induces apoptosis in lung cancer cells dependent on epidermal growth factor receptor signaling for survival. Cancer Res 66:55425548, 2006

8. $\mathrm{Fu} Y \mathrm{~N}, \mathrm{Yeh} \mathrm{CL}$, Cheng $\mathrm{HH}$, et al: EGFR mutants found in non-small cell lung cancer show different levels of sensitivity to suppression of Src: Implications in targeting therapy. Oncogene 27:957965, 2008 
9. Chung BM, Dimri M, George M, et al: The role of cooperativity with $\mathrm{Src}$ in oncogenic transformation mediated by non-small cell lung cancerassociated EGF receptor mutants. Oncogene 28 : 1821-1832, 2009

10. Leung EL, Tam IY, Tin VP, et al: SRC promotes survival and invasion of lung cancers with epidermal growth factor receptor abnormalities and is a potential candidate for molecular-targeted therapy. Mol Cancer Res 7:923-932, 2009

11. Cabioglu N, Summy J, Miller $C$, et al: CXCL12/stromal cell-derived factor-1alpha transactivates HER2-neu in breast cancer cells by a novel pathway involving Src kinase activation. Cancer Res 65:64936497, 2005

12. Pal S, Datta K, Mukhopadhyay D: Central role of p53 on regulation of vascular permeability factor/ vascular endothelial growth factor (VPFNEGF) expression in mammary carcinoma. Cancer Res 61: 6952-6957, 2001

13. Sounni NE, Roghi $C$, Chabottaux $V$, et al: Upregulation of vascular endothelial growth factor-A by active membrane-type 1 matrix metalloproteinase through activation of Src-tyrosine kinases. J Biol Chem 279:13564-13574, 2004

14. Sato M, Tanaka $T$, Maeno $T$, et al: Inducible expression of endothelial PAS domain protein-1 by hypoxia in human lung adenocarcinoma A549 cells: Role of Src family kinases-dependent pathway. Am J Respir Cell Mol Biol 26:127-134, 2002

15. Johnson FM, Agrawal $S$, Burris $H$, et al: Phase I pharmacokinetic and drug-interaction study of dasatinib in patients with advanced solid tumors. Cancer 116:1582-1591, 2009

16. Quintás-Cardama $A$, Kantarjian $H$, O'brien $S$, et al: Pleural effusion in patients with chronic myelogenous leukemia treated with dasatinib after imatinib failure. J Clin Oncol 25:3908-3914, 2007

17. Miller AA, Pang $H$, Hodgson $L$, et al: A phase II study of dasatinib in patients with chemosensitive relapsed small cell lung cancer (Cancer and Leukemia Group B 30602). J Thorac Oncol 5:380-384, 2010

18. Therasse P, Arbuck SG, Eisenhauer EA, et al: New guidelines to evaluate the response to treatment in solid tumors: European Organization for Research and Treatment of Cancer, National Cancer
Institute of the United States, National Cancer Institute of Canada. J Natl Cancer Inst 92:205-216, 2000

19. Wahl RL, Jacene $H$, Kasamon $Y$, et al: From RECIST to PERCIST: Evolving considerations for PET response criteria in solid tumors. J Nucl Med 50:122S-150S, 2009 (suppl 1)

20. Demetri GD, Lo Russo P, MacPherson IR, et al: Phase I dose-escalation and pharmacokinetic study of dasatinib in patients with advanced solid tumors. Clin Cancer Res 15:6232-6240, 2009

21. Haura EB, Tanvetyanon $T$, Chiappori $A$, et al: Phase $\mid / I I$ study of the Src inhibitor dasatinib in combination with erlotinib in advanced non-smallcell lung cancer. J Clin Oncol 28:1387-1394, 2010

22. Schiller JH, Harrington D, Belani CP, et al: Comparison of four chemotherapy regimens for advanced non-small-cell lung cancer. N Engl J Med 346:92-98, 2002

23. Sandler A, Gray R, Perry MC, et al: Paclitaxelcarboplatin alone or with bevacizumab for non-smallcell lung cancer. N Engl J Med 355:2542-2550, 2006

24. Mok TS, Wu YL, Thongprasert $S$, et al: Gefitinib or carboplatin-paclitaxel in pulmonary adenocarcinoma. N Engl J Med 361:947-957, 2009

25. Sen B, Saigal B, Parikh N, et al: Sustained Src inhibition results in signal transducer and activator of transcription 3 (STAT3) activation and cancer cell survival via altered Janus-activated kinase-STAT3 binding. Cancer Res 69:1958-1965, 2009

26. Li J, Rix U, Fang B, et al: A chemical and phosphoproteomic characterization of dasatinib action in lung cancer. Nat Chem Biol 6:291-299, 2010

27. Sos ML, Michel K, Zander $T$, et al: Predicting drug susceptibility of non-small cell lung cancers based on genetic lesions. J Clin Invest 119:17271740, 2009

28. McDermott $U$, Ames RY, lafrate $A J$, et al: Ligand-dependent platelet-derived growth factor receptor (PDGFR)-alpha activation sensitizes rare lung cancer and sarcoma cells to PDGFR kinase inhibitors. Cancer Res 69:3937-3946, 2009

29. Braiteh F, Kurzrock R: Uncommon tumors and exceptional therapies: Paradox or paradigm? Mol Cancer Ther 6:1175-1179, 2007

30. Curtin JA, Busam K, Pinkel D, et al: Somatic activation of KIT in distinct subtypes of melanoma. J Clin Oncol 24:4340-4346, 2006
31. Yaren A, Oztop I, Kargi A, et al: Bax, bcl-2 and c-kit expression in non-small-cell lung cancer and their effects on prognosis. Int J Clin Pract 60:675682, 2006

32. Pietsch $T$, Nicotra MR, Fraioli $R$, et al: Expression of the c-Kit receptor and its ligand SCF in non-small-cell lung carcinomas. Int J Cancer 75:171175, 1998

33. Schmid K, Oehl N, Wrba F, et al: EGFR/KRAS/ BRAF mutations in primary lung adenocarcinomas and corresponding locoregional lymph node metastases. Clin Cancer Res 15:4554-4560, 2009

34. Spruessel A, Steimann G, Jung M, et al: Tissue ischemia time affects gene and protein expression patterns within minutes following surgical tumor excision. Biotechniques 36:10301037, 2004

35. Blackhall FH, Pintilie M, Wigle DA, et al: Stability and heterogeneity of expression profiles in lung cancer specimens harvested following surgical resection. Neoplasia 6:761-767, 2004

36. Mager SR, Oomen MH, Morente MM, et al: Standard operating procedure for the collection of fresh frozen tissue samples. Eur J Cancer 43:828834, 2007

37. Kopetz S, Lesslie DP, Dallas NA, et al: Synergistic activity of the SRC family kinase inhibitor dasatinib and oxaliplatin in colon carcinoma cells is mediated by oxidative stress. Cancer Res 69:38423849, 2009

38. Ceppi $P$, Papotti M, Monica V, et al: Effects of Src kinase inhibition induced by dasatinib in nonsmall cell lung cancer cell lines treated with cisplatin. Mol Cancer Ther 8:3066-3074, 2009

39. Raju U, Johnson FM, Glisson BS, et al: Mechanisms of enhanced radiosensitivity of human head and neck squamous cell carcinomas by dasatinib (BMS-354825, an inhibitor of Src kinases) include induction of apoptosis and inhibition of DNA repair, Proceedings of the American Association for Cancer Research, San Diego, CA, 2008, pp 642

40. Wheeler $D L$, lida $M$, Kruser TJ, et al: Epidermal growth factor receptor cooperates with Src family kinases in acquired resistance to cetuximab. Cancer Biol Ther 8:696-703, 2009 